\title{
Compressible Turbulent Boundary Layers With Heat Transfer and Pressure Gradient in Flow Direction $^{1}$
}

\author{
Alfred Walz
}

(March 11, 1959)

\begin{abstract}
The best-known theoretical works on boundary-layer problems, especially in the case of compressible flow without or with heat transfer, are related to the laminar boundary layer, although the turbulent boundary layer is, in practice, often more interesting. The laminar boundary layer is more easily accessible to theoretical treatment because clearly defined relations exist between the viscosity $\mu$ and the shear stresses $\tau$. In the turbulent ease, empirical relations must be introduced. Therefore, attempts to get exact solutions are not worthwhile, while efforts to obtain approximate solutions, based for instance on the von Karman-Pohlhausen principle (Z. Angew. Math. u. Mech. 1, 233 and 252, 1921) of utilizing integral conditions, appear to be appropriate to this problem. In the last few years the accuracy of such approximate solutions for the incompressible case was noticeably improved by the application of a new energy integral condition in connection with a new empirical law for the dissipation in turbulent boundary layers, stated by J. Rotta (Ingr-Arch. 20, 195, 1952) and E. Truckenbrodt (Ingr-Arch. 20, 212, 1952). The empirical laws for dissipation and for turbulent wall friction, which are needed in the present approximate theory, are formulated on the basis of available measurements for incompressible flow. Generalization to the compressible flow with heat transfer is made from physical considerations. Calculated results agree satisfactorily with available experimental data. Some possibilities for improving as well as for simplifying the approximation theory are outlined.
\end{abstract}

\section{Introduction}

In principle, problems of compressible laminar boundary layers may be solved by using the partial differential equations for the velocity and temperature fields, and the thermodynamic relations between viscosity $\mu$, density $\rho$, pressure $p$, and temperature $T$. Exact solutions for special cases have been worked out by several authors $[5,6,7,8] .{ }^{2}$ Nevertheless, approximate theories of the von Karman-Pohlhausen type have been successful because of their easy adaptability to the most general case of flows with arbitrary pressure gradient in flow direction [9 through 16].

An example is the case of a compressible turbulent boundary layer, which is more important in practice because the controlling viscosity is no longer a material property, such as the molecular viscosity, $\mu$; rather, it is like so-called "apparent viscosity," $\mu_{a}$, which is essentially determined by the empirical laws of turbulent mixing motions. In view of the empirical character of these laws, attempts of exact solutions of Prandtl's boundary layer equations are indeed inappropriate. Hence, approximation theories of the von Karman-Pohlhausen type are indeed the only ones which are well adapted to the problem of calculating turbulent boundary layers.

The first solution was given by E. Gruschwitz [17] in 1931. He introduced a one-parameter family of empirical turbulent velocity profiles with parameter $H$, and, as the second parameter of the problem, the boundary-layer momentum-loss thickness $\delta_{2}$. Gruschwitz then derived an empirical energy law. This was used together with the known von Karman integral condition for the momentum within the boundary layer, to determine the two parameters $H$ and $\delta_{2}$ as functions of $x$ for a given presssure gradient.

The so-called Gruschwitz energy law could not be verified in a general manner and appears therefore somewhat doubtful. Moreover, it is restricted to the incompressible case. A definite improvement of the approximate theory for turbulent boundary layers was accomplished

\footnotetext{
1 This work was supported by the U.S. Air Force, through the Office of Scientific Research of the Air Research and Development Command
}

2 Figures in brackets indicate the literature references at the end of this paper. 
when several authors [9 through 15] independently (between 1935 and 1946) showed that an integral condition of the energy within the boundary layer may be derived from the Prandtl boundary-layer equation in the similar way as for the momentum. Some years later (1950) J. Rotta [3] derived from measurements the empirical law for the dissipation within the turbulent boundary layer, which was needed for the applications of the new integral energy condition. At about the same time the empirical law for the turbulent wall friction occurring in the momentum condition was improved by new accurate measurements of Ludwieg and Tillmann [18], such that it may be generally valid for positive and negative pressure gradients in flow direction.

Now, on the basis of the momentum and energy integral conditions on the one hand and the two empirical laws for the turbulent wall friction and dissipation on the other hand, a two-variable method approximate theory of boundary layer could be developed, similar to that formerly worked out by several authors for laminar boundary layers [9 through 16].

For the incompressible case, the integration method of E. Truckenbrodt [4] (1952) (similar to that developed by A. Walz [19] in 1941 for the laminar case) appears to be the simplest. However, applications to compressible turbulent boundary layer failed, because the semiempirical laws for wall friction and dissipation could not be set up, since before that time measurements of turbulent boundary layers at high Mach numbers were not available in sufficient generality.

Basing his analysis upon simple physical facts about the turbulence mechanism the author was able to derive a generalization of the empirical laws for wall friction and dissipation to include compressibility, $[26,27]$.

The inclusion of the case of heat transfer, however, implies a new difficulty insofar as the integral energy condition derived from Prandtl's equation considers only an energy flux along the body surface but does not relate to an energy flux through the wall. In the latter general case we must start from the general differential equation for the temperature field. In addition it must be checked whether the above compressible considerations would include heat transfer at the wall.

As suggested by L. E. Kalikhman [13] for the case of laminar boundary layers the partial differential equation for the temperature field can be transformed by partial integrations into an infinite system of ordinary differential equations similar to that obtained by partial integration of Prandtl's boundary layer equation.

Hence, two infinite systems of ordinary differential equations with a twice infinite number of unknowns are available which appear to be completely equivalent to the two partial differential equations for the velocity and temperature field.

Some general properties of these systems, which had not yet been outlined in the literature, will be discussed.

As a first step of evaluation of the possibilities involved in these systems of equations, a "two-equation, two-unknown" method is investigated. This is about as simple as the known methods for the incompressible case [4, 19]. Nevertheless results obtained are of sufficient accuracy for engineering purposes.

The application of the new method is illustrated in some examples. Directions for improvement as well as for further simplifications are indicated.

\section{Partial Integration of Prandti's Boundary-Layer Equation for a Compressible Two-Dimensional Steady State Flow Along an Insulated Wall}

\subsection{Derivation of an Infinite System of Ordinary Differential Equations}

As proposed by Leibenson [9], Golubew [10], Sutton [11], K. Weighardt [12], and E. Truckenbrodt [4], Prandtl's system of partial differential equations for a compressible two-dimensional flow along an insulated wall may be transformed into an infinite system of ordinary differential equations by partial integrations. We will give here a new derivation of this 
system which is appropriate for our purposes.

Let $x, y$ be the coordinates, the $x$-direction being taken everywhere tangent to the wall. Further $u$ and $v$ are the components of the velocity vector, respecting in the $x$ and $y$ directions, $\mu$ the viscosity coefficient, $\delta$ the density coefficient. Then Prandtl's boundary-layer equations may be written as:

$$
\begin{gathered}
\rho u \frac{\partial u}{\partial x}+\rho v \frac{\partial u}{\partial y}=\rho_{\delta} u_{\delta} \frac{d u_{\delta}}{d x}+\frac{\partial\left(\mu \frac{\partial u}{\partial y}\right)}{\partial y}, \\
\frac{\partial(\rho u)}{\partial x}+\frac{\partial(\rho v)}{\partial y}=0 \quad \text { (continuity equation). }
\end{gathered}
$$

Here the subscript $\delta$ denotes that the quantity is referred to the edge of the boundary layer. We note that this system is valid even for turbulent boundary layers, if the velocities $u$ and $v$ as well as $\rho$ are considered to be the mean values over time and if $\mu$ is the effective value $\mu \equiv \mu_{e}$, which consists of the molecular viscosity $\mu$, the apparent viscosity $\mu_{a}$. Thus,

$$
\mu_{e}=\mu+\mu_{a} .
$$

For simplicity, however, we prefer to use in both cases, laminar and turbulent, the same symbols $u, v, \rho$, and $\mu$, knowing that in the turbulent case we have $\mu \equiv \mu_{e}$.

First we multiply eq (1) by $u^{\nu}$ and add to it eq (2) multiplied by $u^{\nu+1} /(\nu+1)$.

Partial integration from $y=0$ to $y=\infty$ then yields after elementary transformations,

$$
\frac{1}{\rho_{\delta} u_{\delta}^{\nu+2}} \frac{d}{d x}\left(\rho_{\delta} u_{\delta}^{\nu+2} f_{\nu}\right)+g_{\nu} \frac{d u_{\delta} / d x}{u_{\delta}}+e_{\nu}=0 \quad(\nu=0,1,2, \ldots, \infty) .^{3}
$$

Herein the following notations are used:

$$
\begin{aligned}
& e_{\nu}=(\nu+1) \int_{0}^{u_{\delta}}\left(\frac{u}{u_{\delta}}\right)^{\nu} \frac{\partial}{\partial u}\left(\frac{\tau}{\rho_{\delta} u_{\delta}^{2}}\right) d u, \\
& f_{\nu}=\int_{0}^{\infty}\left[\frac{\rho u}{\rho_{\delta} u_{\delta}}-\frac{\rho}{\rho_{\delta}}\left(\frac{u}{u_{\delta}}\right)^{\nu+2}\right] d y, \\
& g_{\nu}=-(\nu+1) \int_{0}^{\infty}\left[\frac{\rho u}{\rho_{\delta} u_{\delta}}-\left(\frac{u}{u_{\delta}}\right)^{\nu}\right] d y, \\
& \tau=\mu \frac{\partial u}{\partial y} .
\end{aligned}
$$

From the structure of the integral terms (5) through (7) we see that for practical purposes it will not be necessary to extend the integration process beyond a certain distance $y=\delta$, for which we have $u=u_{\delta}, \rho=\rho_{\delta}, \tau=0$, because for $y>\delta$ all integrands became practically zero. The layer thickness $\delta$ is somewhat vague in view of the asymptotic behavior of $u(y)$ at the outer edge. For approximate theories, however, it is very useful and may be defined as wall distance $y$ at which $u$ reaches the value $u_{\infty}$ with an accuracy better than 99 percent. Hence we put

$$
0.99 u_{\infty}<u_{\delta}<u_{\infty}
$$

\subsection{Discussion of the Infinite System of Equations}

Putting $\nu=0$ we obtain the usual momentum integral condition of von Karman-Pohlhausen, for $\nu=1$ we have Weighardt's energy integral condition. Only these two cases have been considered up to the present, but it is clear that all other remaining integral conditions of the

\footnotetext{
${ }^{3} \nu$ may have also values between $-\infty$ and $+\infty$, even nonintegers as $0.1,0.2, \ldots$ and so on.
} 
infinite system (4), the so-called higher momentum conditions $(\nu=2,3,4, \ldots, \infty)$, would be important too, since only the complete system is equivalent to Prandtl's equations.

Let us first investigate the meaning of the equations of order $\nu$, higher than unity.

First eq (4) is rewritten in a more convenient form. At the outer edge $y=\delta$ of the boundary layer, we may assume adiabatic conditions of state. According to the basic laws of gas dynamics we have between the density of $\rho_{\delta}$, the velocity $u_{\delta}$, and the Mach number $M_{\delta}=u_{\delta} / \alpha_{\delta}$ $\left(a_{\delta}=\right.$ local sound velocity) the following relations (see, for instance, R. Sauer [20]):

$$
\frac{1}{\rho \delta} \frac{d \delta}{d x}=-\frac{1}{u \delta} \frac{d u_{\delta}}{d x} M_{\delta}^{2}
$$

Substitution of (10) into (4) gives

$$
f_{\nu}^{\prime}+\frac{u_{\delta}^{\prime}}{u_{\delta}} f_{\nu}\left[2+\nu-M_{\delta}^{2}+\frac{g_{\nu}}{f_{\nu}}\right]+e_{\nu}=0
$$

where the prime denotes differentiation with respect to $x$.

The integral terms $e_{\nu}, f_{\nu}, g_{\nu}$ represent the unknowns of the system. They contain the velocity and density distributions $u(x, y) / u_{\delta}(x)$ and $\rho(x, y) / \rho_{\delta}(x)$, respectively, which are indeed the real unknowns of the problem.

The only way to obtain a solution of the problem was to introduce approximations for $u / u_{\delta}$ and $\rho / \rho_{\delta}$ according to von Karman-Pohlhausen's idea, for instance by means of a oneparameter family of experimental curves. In the incompressible case $\left(\rho / \rho_{\delta}=1\right)$ the problem was indeed solved in this way by taking two equations from system (11) (for $\nu=0$ and $\nu=1$ ), ${ }^{4}$ using as unknowns the boundary layer thickness $\delta$ or one of the functions $e_{\nu}, f_{\nu}, g_{\nu}$, and, as the second unknown, a shape parameter $H$ of the velocity profile. In this case the ratios $e_{\nu} / \delta$, $f_{\nu} / \delta, g \nu / \delta$, as well as $e_{\nu} / f_{\nu}$ and $g_{\nu} / f_{\nu}$, are only functions of the shape parameter $H$.

The compressible case involves new variables $\rho$ and $T$ which are related by

$$
\frac{\rho}{\rho_{\delta}}=\frac{T_{\delta}}{T}
$$

since $\partial p / \partial y=0$. Additional equations will be needed to make the problem determinate. This is done for the thermal boundary layer. While we will return to this problem in section 3 of this report, let us first discuss the principal properties of the infinite system (11).

The order number $\nu$ may for instance be increased toward $\infty$. In this case we have from equations (6) and (7),

and

$$
\left[f_{\nu}\left(\nu+2-M_{\delta}^{2}\right)+g_{\nu}\right]_{\nu=\infty}=f_{\infty}\left(1-M_{\delta}^{2}\right),
$$

becanse

$$
f_{\infty}=\int_{0}^{\delta} \frac{\rho u}{\rho_{\delta} u_{\delta}} d y
$$

$$
\left(\frac{u}{u_{\delta}}\right)_{\nu \rightarrow \infty}^{\nu+2}=\left(\frac{u}{u_{\delta}}\right)_{\nu \rightarrow \infty}^{\nu}=0, \quad \text { for } 0<\frac{u}{u_{\delta}}<1 \text {, }
$$

\footnotetext{
4 The original Pohlhausen method only used the equation $\nu=0$ (momentum equation) from this system. As the second equation, Pohlhausen introduced the so-called wall condition,

$$
\left(\mu \frac{\partial^{2} u}{\partial y^{2}}\right)_{y=0}=\frac{d p}{d x}
$$

which follows directly from Prandtl's eq (1) for the point $y=0$. It is obvious that this condition, valid for the wall point, would be doubtful if used for fixing the shape parameter. Indeed essential improvements of results were observed when this wall condition was neglected and replaced by the equation for $\nu=1$ (see, for instance, [16]).
} 
whatever the functions $u / u_{\delta}$ and $\rho / \rho_{\delta}$ may be in detail.

The integral expression $\left(e_{\nu}\right)_{\nu \rightarrow \infty}$ is found to be zero as follows:

For $0<\frac{u}{u_{\delta}}<1$ it yields

$$
\left[(1+\nu)\left(\frac{u}{u_{\delta}}\right)^{\nu}\right]_{\nu \rightarrow \infty} \rightarrow 0 .
$$

Since furthermore the shear stress $\tau$ and its derivative $\partial \tau / \partial y$ are vanishing at $y=\delta\left(u=u_{\delta}\right)$ for arbitrary velocity profile $u / u_{\delta}$, we have

$$
\left(\mathrm{e}_{\nu}\right)_{\nu \rightarrow \infty} \rightarrow 0 \text {. }
$$

Hence in the limiting case of $\nu=\infty$, system (11) reduces to

$$
f_{\infty}^{\prime}+f_{\infty}\left(1-M_{\delta}^{2}\right) \frac{u_{\delta}^{\prime}}{u_{\delta}}=0 .
$$

This equation may be called the mass-flow equation and is nothing less than an averaged form of the continuity equation. With the aid of (10) it can immediately be integrated to

where

$$
f_{\infty} \rho_{\delta} u_{\delta}=\left(\delta-\delta_{1}\right) \rho_{\delta} u_{\delta}=\text { const. }
$$

$$
\delta_{1}=\int_{0}^{\delta}\left(1-\frac{\rho u}{\rho_{\delta} u_{\delta}}\right) d y
$$

is known as the displacement thickness. This relation has not previously been used in approximate boundary layer theories. It expresses that the mass flow between the stream lines $y=\delta$ and $y=\delta_{1}$ must be constant. We have not ascertained whether relation (19) will be very useful in practice. But it is clear that if the velocity and density profiles are once determined in the inner part of the boundary layer (thereby determining the value $\delta_{1}$ ), the total boundary layer thickness $\delta$ is determined also by relation (19).

We have found that if the two-equation system $\nu=0, \nu=1$ with two unknowns is used, this relation is mostly violated. Nevertheless the essential properties of the boundary layer, for example, wall friction and separation behavior, appear to be correctly described.

From this consideration about the mass-flow equation as limiting case for $\nu=\infty$, we may learn that the higher the order $\nu$, the less details of the velocity profile will be included in the corresponding equation, because the term $e_{v}$, which contains the shearing stress (and with this the entire boundary layer effect), approaches zero, with $\nu \rightarrow \infty$.

It is therefore not surprising that equations corresponding to low order, for example the equation for $\nu=0$ and $\nu=1$, still give a very good description of the properties of the boundary layer if a rather physically reasonable family of velocity profiles is introduced into the equations. A good way in principle as suggested for the incompressible laminar boundary layers a long time ago by several authors $[9,10,11,12]$, would be to restrict to a finite number of equations of the system, preferably to those of low order, say up to $\nu=5$ or 10 , and to introduce a polynomial expression for the velocity profile

$$
\frac{u}{u_{\delta}}=\frac{u}{u_{\delta}}\left[\frac{y}{\delta_{2}(x)}, H_{1}(x), H_{2}(x), H_{3}(x) \ldots, H_{\nu}(x)\right]
$$

with $\nu$ coefficients $H$. Then $\nu+1$ ordinary differential equations will allow the determination of the $\nu$ coefficients $H_{\nu}$ and $\delta_{2}$ as functions of $x$. In this case no detailed assumption about the velocity profile, except some trivial boundary conditions, must be made.

It appears worthwhile to study this problem with new efforts, because its solution will be to a large extent equivalent to the solution of Prar.dtl's boundary layer equation, but needs no restrictions as to the prescribed pressure distribution $p(x)$, and will in principle be applicable to the more important case of turbulent boundary layers too. Since we want to include the 
compressible case with heat transfer at the wall, we must simultaneously consider the temperature boundary layer.

The infinite system of equations derived from Prandtl's equation contains no terms which take into account an energy flux through the wall. Therefore, only the momentum equation $\nu=0$ and the mass-flow equation $\nu=\infty$ of this system can be used in connection with heat transfer problems. All other equations are worthless in this respect. The essential step to get out of this difficulty is to include the partial differential equation for the temperature field in this problem by transforming it into an infinite system of ordinary differential equations similar to that obtained from Prandtl's equation.

\section{Derivation of Integral Conditions From the Partial Differential Equation for the Temperature Field}

The general partial differential equation for the temperature field in a two-dimensional steady state flow may be written as follows, if the enthalpy $i=c_{p} T$ is introduced:

$$
\rho u \frac{\partial i}{\partial x}+\rho v \frac{\partial i}{\partial y}=u \frac{d p}{d x}+\mu\left(\frac{\partial u}{\partial y}\right)^{2}+\frac{\partial}{\partial y}\left(\frac{\lambda}{c_{p}} \frac{\partial i}{\partial y}\right) .
$$

Here $\lambda$ denotes the heat conductivity ( $\mathrm{kcal} / \mathrm{m} \mathrm{sec}{ }^{0} \mathrm{~K}$ ), $c_{p}$ the specific heat at constant pressure, $u, v$ must satisfy the continuity equation

$$
\frac{\partial(\rho u)}{\partial x}+\frac{\partial(\rho v)}{\partial y}=0
$$

Now, if we multiply eq (22) by $i$, and eq (23) by $i^{\nu+1} / \nu+1$ and add the multiplied equations, we obtain

$$
\frac{\partial\left(\rho u i^{\nu+1}\right)}{\partial x}+\frac{\partial\left(\rho v i^{\nu+1}\right)}{\partial y}=(\nu+1)\left\{-u \rho_{\delta} u_{\delta} \frac{d u_{\delta}}{d x} i^{\nu}+\mu\left(\frac{\partial u}{\partial y}\right)^{2} i^{\nu}+\frac{\partial}{\partial y}\left(\frac{\lambda}{c_{p}} \frac{\partial i}{\partial y}\right) i^{\nu}\right\} .
$$

By partial integration of eq (24) with respect to $y$ from $y=0$ to $y=\infty$ and by substitution for $v$ from eq $(23)$ we have

$$
\begin{aligned}
& \frac{d}{d x}\left[\int_{0}^{\infty}\left(\rho u i^{\nu+1}-\rho_{\delta} u_{\delta} i_{\delta}^{\nu+1}\right) d y\right]+\frac{d}{d x}\left[\left(\rho_{\delta} u_{\delta} i_{\delta}^{\nu+1}\right) \delta\right]-i_{\delta}^{\nu+1} \frac{d}{d x}\left[\int_{0}^{\infty}\left(\rho u-\rho_{\delta} u_{\delta}\right) d y\right]-i_{\delta}^{\nu+1} \frac{d}{d x}\left[\rho_{\delta} u_{\delta} \delta\right] \\
& \quad=(\nu+1)\left\{-\rho_{\delta} u_{\delta} \frac{d u_{\delta}}{d x} \int_{0}^{\infty}\left(u i^{\nu}-u_{\delta} i_{\delta}^{\nu}\right) d y-\rho_{\delta} u_{\delta} \frac{d u_{\delta}}{d x} u_{\delta} i_{\delta}^{\nu} \delta+\int_{0}^{\delta} i^{\nu} d\left[\frac{\lambda}{c_{p}} \frac{\partial i}{\partial y}\right]+\int_{0}^{\infty} \mu i^{\nu}\left(\frac{\partial u}{\partial i}\right)^{2} d y\right\} .
\end{aligned}
$$

The terms with the factor $\delta$ vanish because of the following known relation in gas dynamics:

$$
\frac{1}{i_{\delta}} \frac{d i_{\delta}}{d x}+\frac{u_{\delta}^{2}}{i_{\delta}} \frac{1}{u_{\delta}} \frac{d u_{\delta}}{d x}=0
$$

which follows after a short intermediate calculation from

$$
\frac{u_{\delta}^{2}}{i_{\delta}}=(k-1) M_{\delta}^{2}, \quad k=\frac{c_{p}}{c_{v}},
$$

and

$$
M_{\delta}^{2}=\frac{2}{k+1} \frac{M_{\delta}^{*^{2}}}{1-\frac{k-1}{k-1} M_{\delta}^{*^{2}}} ; \quad M_{\delta}^{*}=\frac{u_{\delta}}{a^{*}} ; \quad a^{*}=\text { critical sound velocity }
$$




$$
\frac{1}{M_{\delta}} \frac{d M_{\delta}}{d x}=\frac{1}{u_{\delta}} \frac{d u_{\delta}}{d x}\left(1+\frac{k-1}{2} M_{\delta}^{2}\right)
$$

Using notations for the integral terms:

$$
\int_{0}^{\infty} \frac{\rho u}{\rho_{\delta} u_{\delta}}\left[\left(1-\frac{\rho_{\delta}}{\rho}\right)^{\nu+1}\right] d y \approx \int_{0}^{\delta} \frac{\rho u}{\rho_{\delta} u_{\delta}}\left[1-\left(\frac{\rho_{\delta}}{\rho}\right)^{\nu+1}\right] d y=\phi_{\nu}
$$

( $\infty$ can be replaced by $\delta$, because the integrand is almost vanishing for $y \geqq \delta$ )

with

$$
\begin{gathered}
\int_{0}^{1}\left(\frac{\rho_{\delta}}{\rho}\right)^{\nu} \frac{\tau}{\tau w} d\left(\frac{u}{u_{\delta}}\right)=\epsilon_{\nu}, \\
\int_{0}^{u_{\delta}}\left(\frac{\rho_{\delta}}{\rho}\right)^{\nu} \frac{\partial}{\partial u}\left[\frac{\tau}{\tau_{w}} \frac{\partial\left(T / T_{\delta}\right)}{\partial\left(u / u_{\delta}\right)}\right] d u=\gamma_{\nu},
\end{gathered}
$$

and

$$
\tau=\mu \frac{\partial u}{\partial y}, \tau_{w}=\left[\mu \frac{\partial u}{\partial y}\right]_{y=0}, \frac{\mu c_{p}}{\lambda}=P_{r}=\text { constant Prandtl number },
$$

$$
\frac{T}{T \delta}=\frac{i}{i_{\delta}}=\frac{\rho_{\delta}}{\rho}=\left(\text { because } \frac{\partial p}{\partial y}=0\right),
$$

equation (25) may be rewritten as follows:

$$
\frac{d \phi_{\nu}}{d x}+\phi_{\nu} \frac{d u_{\delta}}{d x} \frac{1-M_{\delta}^{2}}{u_{\delta}}=-(\nu+1) \frac{k-1}{2} M_{\delta}^{2} \frac{\tau_{w}}{\rho_{\delta} u_{\delta}^{2}}\left\{2 \epsilon_{\nu}+\frac{\gamma_{\nu}}{P_{r} \frac{k-1}{2} M_{\delta}^{2}}\right\} .
$$

$\phi_{\nu}, \epsilon_{\nu}$ and $\gamma_{\nu}$ (with $\nu$ as an arbitrary number) are an infinite number of unknowns in this infinite system of ordinary differential equations. The real unknowns, however, are the distributions $u(x, y) / u_{\delta}(x)$ and $T(x, y) / T_{\delta}(x)$ which we are trying to determine by means of the infinite number of integral conditions as expressed in the system (35). Now, before going to the solution, let us transform it into a somewhat more convenient form. First a new variable is introduced:

$$
\eta_{\nu}=\frac{\phi_{\nu}}{\delta_{2}(k-1) M_{\delta}^{2}}
$$

where $\delta_{2}$ is the displacement thickness, defined by

$$
\delta_{2}=\int_{0}^{\delta} \frac{\rho u}{\rho_{\delta} u_{\delta}}\left(1-\frac{u}{u_{\delta}}\right) d y=f_{0} \quad(\text { see }(11)) .
$$

It is to be remembered that $\eta_{\nu}$ is finite when $M_{\delta} \rightarrow 0$, while $\phi_{\nu}$ has the disadvantage of becoming zero when $M_{\delta} \rightarrow 0$. Furthermore,

$$
\begin{aligned}
& \Phi=1+(k-1) M_{\delta}^{2}-\frac{\delta_{1}}{\delta_{2}}, \quad \delta_{1}=\int_{0}^{\delta}\left(1-\frac{\rho u}{\rho_{\delta} u_{\delta}}\right) d y, \\
& \Psi_{\nu}=(\nu+1) \frac{\tau_{w}}{\rho_{\delta} u_{\delta}^{2}}\left\{\epsilon_{\nu}+\frac{\gamma_{\nu}}{P_{r}(k-1) M_{v}^{2}}+\frac{\eta_{\nu}}{\nu+1}\right\} .
\end{aligned}
$$

With those notations (35) can be rewritten as follows:

$$
\frac{d \eta_{\nu}}{d x}+\eta_{\nu} \Phi \frac{d^{d} u_{\delta} / d x}{u_{\delta}}+\frac{\Psi_{\nu}}{\delta^{2}}=0 \quad(\nu=0,1,2, \ldots, \infty) .
$$


In principle a possible way to solve the problem of determining the distribution $u(x, y) / u_{\varepsilon}(x)$ and $\rho(x, y) / \rho_{\delta}(x)$ is to use polynomial expressions which fulfill the boundary conditions of the velocity and thermal boundary layer, by choosing $j$ coefficients which satisfy $j$ equations of the system (40). It is obvious that in the present case the procedure is more complicated than in the case of system (11). However, it may be possible to overcome by taking the solution from the exact theory for the temperature profile $T / T_{\delta}$ or the density profile $\rho / \rho_{\delta}=T_{\delta} / T$. Although the solution is exactly valid only for the special case of the flat plate $(d p / d x=0)$, it may be considered as a good approximation even for the more general case with $d p / d x \neq 0$ (see for instance H. Schlichting [21, p. 282]). An essential property of this solution for $T / T_{\delta}$ is that it depends not upon $x$ but only upon $u / u_{\delta}$ with the Mach numbers $M_{\delta}$ and the heat transfer coefficient $c_{T}$ as parameters.

With reference to a paper of von Driest [23] this solution for $T / T_{\delta}$ for the case $d p / d x=0$ can be written as

$$
\frac{T}{T_{\delta}}=1+c_{T}\left(f_{1}-1\right)+r \frac{k-1}{2} M_{\delta}^{2}\left(1-f_{2}\right)
$$

with

$$
\begin{gathered}
f_{1}\left(\frac{u}{u_{\delta}}, P_{r}\right) \approx \frac{u}{u_{\delta}}, \quad f_{2}\left(\frac{u}{u_{\delta}}, P_{r}\right) \approx\left(\frac{u}{u_{\delta}}\right)^{2} \text { for } 0.8<P_{r}<1, \\
r=\frac{T_{r}-T_{\delta}}{T_{0}-T_{\delta}}=\text { recovery factor, } \\
c_{T}=\frac{T_{r}-T_{w}}{T_{\delta}}=\text { heat transfer parameter. }
\end{gathered}
$$

Thus the temperature and density profiles occurring in the integral conditions (36) to (39) are uniquely correlated to the velocity profiles $u / u_{\delta}$, which now are the only unknown functions in the problem. So far all considerations and derivations are valid both for the laminar and turbulent case. For the solution of the laminar case, we have only to introduce an expression for the velocity profiles. The viscosity, as principal parometer of all boundary layer theories is known to be dependent only upon the temperature $T$, for instance according to Sutherland's law.

$$
\frac{\mu}{\mu_{1}}=\frac{T_{1}+C}{T+C}\left(\frac{T}{T_{1}}\right)^{3 / 2}
$$

(with $C=113^{\circ} \mathrm{K}$ for air, $T_{1}$ being a reference temperature) or to its more convenient approximation

$$
\frac{\mu}{\mu_{1}}=\left(\frac{T}{T_{1}}\right)^{\omega}
$$

with

$$
\omega=\frac{3}{2}-\frac{1}{1+C / T_{1}}
$$

( $\omega \approx 0.80$ for temperatures $T$ between $233^{\circ}$ and $373^{\circ} \mathrm{K}, \omega=0.5$ for very high temperatures, $\omega=1.5$ for very small temperatures near the zero point).

In the case of turbulent boundary layers which are mainly considered in this report, an empirical relation for the effective viscosity (as the sum of the apparent and molecular viscosity) must be introduced besides the assumption for the velocity profiles. Before this is done, a simplification of the systems (11) and (40) will now be considered. 


\section{Special Case of a Two-Equation System}

\subsection{General Considerations}

As a first step of evaluation of the possibilities involved in systems (11) and (40) we will make a so-called one-parametric representation for $u / u_{\delta}$, viz:

$$
\frac{u}{u_{\delta}}=\frac{u}{u_{\delta}}\left(\frac{y}{\delta(x)}, \quad H(x)\right)
$$

where $H(x)$ is the form parameter of the velocity profile (as one unknown of the problem) and $\delta(x)$ is the boundary layer thickness (as second unkown). Therefore, to be correct, the relations (48) should be denoted as a two-parametric one.

It must be pointed out that $\delta(x)$ may be replaced by another characteristic length of the boundary layer, preferably by the momentum-loss thickness $\delta_{2}(x)$, thus avoiding any assumption as to the point where $u / u_{\delta}$ reaches the value 1 .

Since we have now decided to use only two unknowns, $H(x)$ and $\delta_{2}(x)$, we need only two equations besides the empirical law for effective viscosity.

We choose the simplest equations (namely those for $\nu=0$ ) from both the system (11) based upon Prandtl's equation (1) and the system (40) based upon the general temperature field equation $(2) .^{5}$

These equations are:

a. The momentum integral condition from system (11). This is the only equation of the system (11) which, besides the mass-flow equation, is valid even in the case with heat transfer at the wall.

$$
f_{0}^{\prime}+f_{0}\left[\frac{g_{0}}{f_{0}}+2-M_{\delta}^{2}\right] \frac{u_{\delta}^{\prime}}{u_{\delta}}+e_{0}=0,
$$

with

$$
\begin{gathered}
e_{0}=-\frac{\tau_{w}}{\rho_{\delta} u_{\delta}^{2}}, \\
f_{0}=\int_{0}^{\delta} \frac{\rho u}{\rho_{\delta} u_{\delta}}\left[1-\frac{u}{u_{\delta}}\right] d y=\delta_{2}, \\
g_{0}=\int_{0}^{\delta}\left(1-\frac{\rho u}{\rho_{\delta} u_{\delta}}\right) d y=\delta_{1} .
\end{gathered}
$$

b. The energy integral condition from system $(40)$

$$
\eta_{0}^{\prime}+\eta_{0} \Phi \frac{u_{\delta}^{\prime}}{u_{\delta}}+\frac{\Psi_{0}}{\delta_{2}}=0
$$

with

$$
\begin{aligned}
\eta_{0} & =-\frac{1}{(k-1) M_{\delta}^{2}} \frac{\int_{0}^{\delta} \frac{u}{u_{\delta}}\left(1-\frac{\rho}{\rho_{\delta}}\right) d y}{\delta_{2}}, \\
\Phi & =1+(k+1) M_{\delta}^{2}-\frac{\delta_{1}}{\delta_{2}} \quad \text { (independent of } \nu \text { ), } \\
\Psi_{0} & =\frac{\tau_{w}}{\rho_{\delta} u_{\delta}^{2}}\left\{\int_{0}^{1} \frac{\tau}{\tau_{w}} d\left(\frac{u}{u_{\delta}}\right)+\eta_{0}-\frac{1}{2} \frac{\theta}{s}\right\},
\end{aligned}
$$

${ }^{5}$ The value of the simple mass-flow equation (19), which may also be obtained from system (40) by putting $\nu=-\infty$, appears to be limited, but has not yet been studied in detail. 


$$
\begin{aligned}
& \theta=\frac{c_{T}}{\frac{k-1}{2} M_{\delta}^{2}}=\frac{T_{r}-T_{w}}{T_{\delta}} \frac{1}{\frac{k-1}{2} M_{\delta}^{2}}, \\
& \left.s \approx P_{r} \int_{0}^{1}\left(\frac{\tau}{\tau_{w}}\right)^{P_{r}-1} d\left(\frac{u}{u_{\delta}}\right) \approx P_{r} \quad \text { (for } P_{r}=0,8 \text { to } 1\right) .
\end{aligned}
$$

For the practical application of the two systems (49) and (53) it was found to be very helpful to evaluate once for all the universal functions $\delta_{1}, \delta_{2}, \eta_{0}, \Phi$, and $\Psi_{0}$ for the interesting range of the parameters $H, M_{\delta}$, and $\theta$ (or $c_{T}$ ). Such an evaluation will be based upon a certain physically reasonable assumption about the velocity profile, as represented by (48). These assumptions for $u / u_{\delta}$ must of course be different in the laminar and turbulent cases. In the turbulent case, the empirical laws for the wall friction $\tau_{w}$ and the dissipation integral $\int_{0}^{u_{\delta}} \tau \partial u$ must also be introduced. The evaluation for the laminar boundary layer contains no difficulties, and will be dealt with in a separate report. Only the more complicated case of the turbulent boundary layer, which needs some special considerations will be treated in this report.

\subsection{Two-Equation Method for Turbulent Boundary Layers}

We now consider: a) A suitable expression for empirical turbulent velocity profiles, b) an empirical law for the wall friction in a compressible turbulent boundary layer with heat transfer, c) an empirical law for the dissipation in a compressible turbulent boundary layer with heat transfer, and d) a method of solution of the system of equations (49) and (53).

\section{a. The Turbulent Velocity Profile}

Detailed investigations of many authors $[23,24,3]$ have shown that the turbulent boundary layer can be divided into several (usually into two) regions with respect to the direction normal to wall, in each one of which different laws for the influence of the outer pressure distribution and the Reynolds number are valid. Thus a general representation for the velocity profile requires a somewhat complicated formula. If only mean properties (integral quantities) of the boundary layer are considered and put into correlation as in our case, it will be sufficient to have an expression which contains less details but includes the whole range of the boundary.

Such a formula, which is proved valid even at high Mach numbers, is

$$
\frac{u}{u_{\delta}}=\frac{y^{k(H)}}{\delta}
$$

where $k$ is the form parameter with values approximately 0.1 and 0.7 , and is a unique function of any $H$ to be chosen.

A disadvantage of (59) is of course that at the wall $y=0$ the value ( $\partial u / \partial y)_{y=0}$ is infinite for all $0<k<1$ and that furthermore, at $y / \delta=1, \partial u / \partial y$ does not vanish as is physically required. But we have shown in previous publication $[26,27]$ that these facts do not essentially influence the integral terms of the approximate theory, since the wall-friction law and the relation between the velocity profile $u / u_{\delta}$ and the shear stress profile $\tau / \tau_{w}$ are separa ${ }^{\ddagger}$ ely introduced as semiempirical laws.

As an example of the suitability of this power law even for Mach numbers up to about 7 and with heat transfer at the wall, we refer to figure 1 which is drawn from experiments of R. E. Wilson [25]. This simple power law is very adequate to the purpose of this approximate theory with regard to the possibility of closed evaluation of the integral terms. Figure 2 shows the order of magnitude of the errors in the ratio $\left(\partial_{3} / \partial_{2}\right)_{i}$ plotted against $\left(\partial_{1} / \partial_{2}\right)_{i}$,

$$
\left(\left(\delta_{3}\right)_{i}=\int_{0}^{\delta} \frac{u}{u_{\delta}}\left[1-\left(\frac{u}{u_{\delta}}\right)^{2}\right] d y\right),
$$




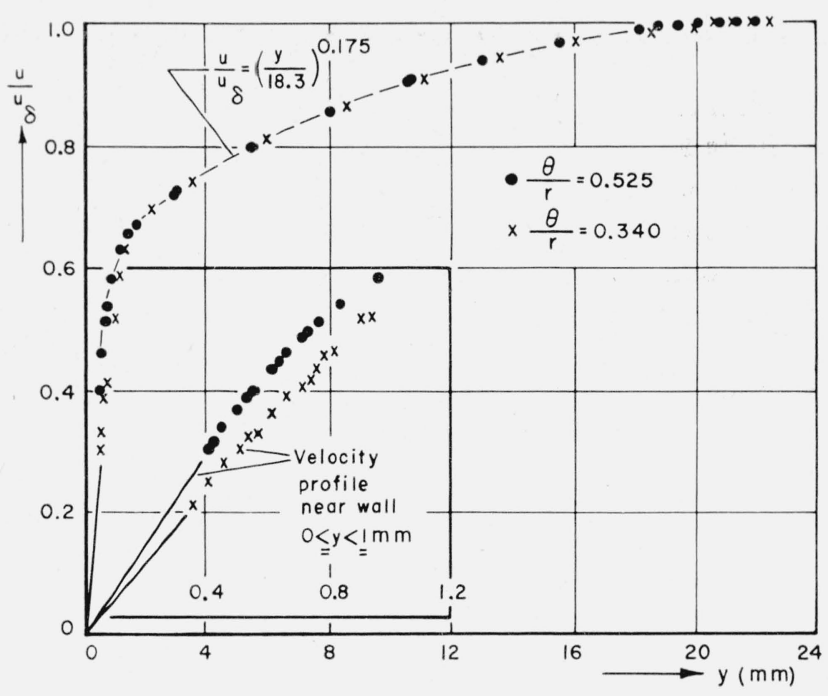

Figure 1. Measured turbulent velocity profiles at a Mach number $M_{\delta}=6.8$ and cooled wall $\left(\frac{\theta}{r}=0.340(\times)\right.$ and $\left.0.525(\bigcirc)\right)$ from R. E. Wilson [25].

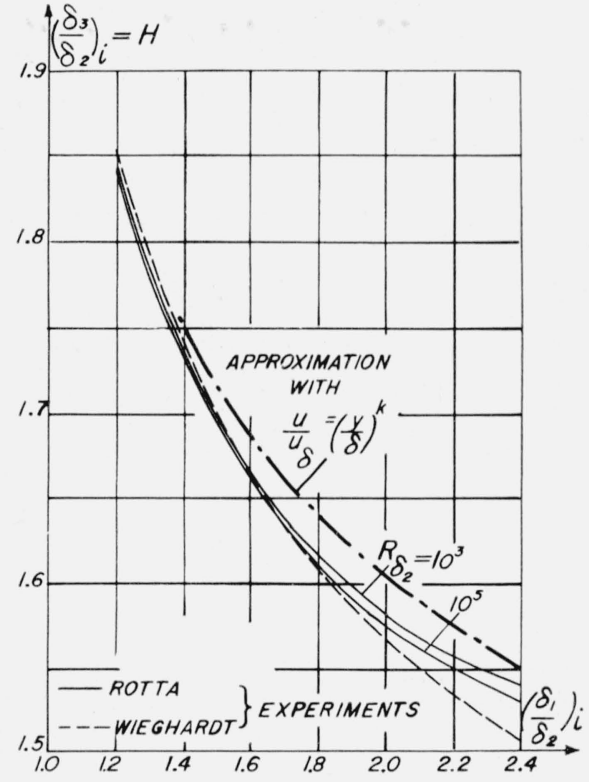

FIGURE 2. Ratio $\left(\delta_{3} / \delta_{2}\right)=H$ plotted against the ratio $\left(\delta_{1} / \delta_{2}\right)$, for turbulent velocity profiles.

Comparison of experiments (according to J. Rotta and K. Weighardt) with approximation power law $\frac{u}{u_{i}}=$ $\left(\frac{y}{\delta}\right) k(H)$.

caused by the approximation of the experimental velocity profiles with the power law calculated for the incompressible case. We see that the maximum error is about 1 percent. The neglect of a certain influence of the Reynolds number on the velocity profile causes errors less than 1 percent. Therefore, the use of the power law (59) for our approximate theory appears to be justified.

Some improvement at the outer edge region of the boundary layer can be reached without much complication by taking the following form:

$$
\frac{u}{u_{\delta}}=\tanh [b \log (1+a y)],
$$

where $a$ and $b$ are two form-parameters, comparable to $H_{1}$ and $H_{2}$. $\quad a$ may be used for the purposes of the adjustment of scales in the one-parametric theory, and the adjustment of wall friction in the two-parametric theory. The relation (60) avoids the use of $\delta$ which is physically vague, and yields automatically an asymptotic behavior near the edge of the boundary layer. At the same time it prevents an infinite slope $\partial u / \partial y$ at $y=0$.

Investigations of the practical importance of expressions (60) (which of course, excludes the possibility of closed integrations) are in progress.

\section{b. The Wall Friction Law}

For this we may refer to the author's publications dealing with the case of compressible turbulent boundary layers without heat transfer $[26,27]$. We now have only to investigate to what extent the relations found there will be influenced by heat transfer at the wall.

As a summary of these earlier investigations we can state that the laws for turbulent mixing motion found for the incompressible case, are valid in the compressible case, provided that the fluctuation velocity is less than the sound velocity, in other words, if the velocity fluctuations are not accompanied by density fluctuations. Now, since the turbulent fluctuation velocity in boundary layers is found to be small, say 10 percent or 20 percent of the main velocity $u_{\delta}$ 
at the outer edge of the boundary layer, $M_{\delta}=\frac{u_{\delta}}{a_{\delta}}$ may be increased to about 10 or 5 , respectively, until the fluctation motion becomes compressible in character. Therefore we conclude that up to about Mach number $M=10$ or 5 , respectively, the laws for incompressible fluctuation may be considered as valid to a first approximation. Only the flow medium properties $\mu$ and $\rho$ are changed. These, however, are taken into account by a suitably chosen Reynolds number.

As to the effect of heat transfer at the wall, this will change only the flow medium properties and therefore can be included in the compressibility effect and in the Reynolds number, which itself plays a decisive role in the wall friction and dissipation law. ${ }^{6}$

The momentum law ( $\nu=0$ from system (11)) contains the term $\tau_{w} / \rho_{\delta} u_{\delta}^{2}$ which is the ratio of the wall friction $\tau_{w}$ to twice the stagnation pressure. This ratio can be rewritten as follows:

$$
\frac{\tau_{w}}{\rho_{\delta} u_{\delta}^{2}}=\frac{\mu_{w}\left(\frac{\partial u}{\partial y}\right)_{w}}{\rho_{\delta} u_{\delta}^{2}}=\frac{\left[\frac{\partial\left(u / u_{\delta}\right)}{\partial y / \delta_{2}}\right]_{w}}{\frac{\rho_{\delta} u_{\delta} \delta_{2}}{\mu_{w}}}=\frac{\left[\frac{\partial\left(u / u_{\delta}\right)}{\partial\left(y /\left(\delta_{2}\right)_{i}\right)}\right]_{w}}{R_{\delta_{2}}} \cdot \frac{\delta_{2}}{\left(\delta_{2}\right)_{i}}\left(H, M_{\delta}, \theta\right), \quad\left(\delta_{2}\right)_{i}=\int_{0}^{\delta} \frac{u}{u_{\delta}}\left(1-\frac{u}{u_{\delta}}\right) d y .
$$

A definition of a Reynolds number

$$
R_{\delta_{2}}=\frac{\rho_{\delta} u_{\delta} \delta_{2}}{\mu_{w}}
$$

is suggested which contains the viscosity $\mu_{w}$ at the wall and, by means of the integral term $\delta_{2}$, an averaged inertia term $\rho_{\hat{\delta}} u_{\delta} \delta_{2}$. Indeed the momentum integral condition (49) brings into relationship the wall friction and the averaged inertia forces. The pressure forces are not of interest in this connection. Any other definition of the Reynolds number appears to be not suitable in the present problem. In the incompressible case these detailed considerations are of course not necessary.

In the numerator of eq (61) we have the dimensionless slope of $u(y)$ at $y=0$. As scale for the length $y$ the value $\left(\delta_{2}\right)_{\text {incompr. }}=\left(\delta_{2}\right)_{i}$ as a wholly geometrical quantity, thus concentrating all influences of Mach number and heat transfer in the universal function $\delta_{2} /\left(\delta_{2}\right)_{i}$. This function is theoretically given, when expressions for $u / u_{\delta}$ and $\rho / \rho_{\delta},(48)$ and (41), are fixed. Next the following term is considered:

$$
\left[\frac{\partial\left(u / u_{\delta}\right)}{\partial\left(y /\left(\delta_{2}\right)_{i}\right.}\right]_{w}{\frac{1}{R_{\delta_{2}}}}=\left(\frac{\tau_{w}}{\rho_{\delta} u_{\delta}^{2}}\right)_{i}
$$

of eq (61) as the wall friction law for incompressible flow. For turbulent boundary layers the slope $\left.\left[\partial\left(u / u_{\delta}\right) / \partial y / \delta_{2}\right)_{i}\right]_{w}$ depends itself on the Reynolds number $R_{\delta 2}$ in such a manner that

$$
\frac{\left[\frac{\partial\left(u / u_{\delta}\right)}{\left(y /\left(\delta_{2}\right)_{i}\right.}\right]_{w}}{R_{\delta_{2}}}=\frac{\alpha(H)}{R_{\delta_{2}}^{n}}=\left(\frac{\tau_{w}}{\rho_{\delta} u_{\delta}^{2}}\right)_{i}
$$

with $n=0.268$ (according to Ludwieg-Tillman [18]) and

$$
\alpha(H)=0.123 \cdot 10^{-0.678\left(\delta_{1} / \delta_{2}\right)_{i}}
$$

as an empirical function, which expresses the influence of shape of $u / u_{\delta}$. Therefore, we can finally write

\footnotetext{
${ }^{6}$ A certain confirmation for these considerations may be seen in figure 1, which shows that the velocity distribution and therefore the related shear stress distribution $\tau / \tau_{w}$ too, is the same as in the incompressible case.
} 


$$
\frac{\tau_{w}}{\rho_{\delta} u_{\hat{\delta}}^{2}}=\frac{\alpha(H)}{R_{\delta_{2}}^{0.268}} \cdot \frac{\delta_{2}}{\left(\delta_{2}\right)_{i}}\left(H, M_{\delta}, \theta\right) .
$$

\section{c. The Dissipation Law}

A generalization similar to that found for the wall friction can be derived for the dissipation in a compressible turbulent flow with heat transfer. The energy integral condition (53) contains the term

$$
\int_{0}^{u_{\delta}} \tau d u=D \text { (dissipation) }
$$

Written in dimensionless form,

$$
\frac{D}{\rho_{\delta} u_{\delta}^{3}}=\frac{\tau_{w}}{\rho_{\delta} u_{\delta}^{2}} \int_{0}^{1} \frac{\tau}{\tau_{w}} d\left(\frac{u}{u_{\delta}}\right)=\left[\left(\frac{\tau_{w}}{\rho_{\delta} u_{\delta}^{2}}\right)_{i} \int_{0}^{1} \frac{\tau}{\tau_{w}} d\left(\frac{u}{u_{\delta}}\right)\right] \frac{\delta_{2}}{\left(\delta_{2}\right)_{i}}
$$

The term $\left(\tau_{w} / \rho_{\delta} u_{\delta}^{2}\right)_{i}$ is given with eq (64). The remaining integral term is found empirically by T. Rotta [3] and E. Truckenbrodt [4] to be

$$
\int_{0}^{1} \frac{\tau}{\tau_{w}} d\left(\frac{u}{u_{\delta}}\right) \approx \frac{\beta(H)}{\alpha(H)} R_{\delta_{2}}^{0.1}
$$

so that it finally yields

$$
\frac{D}{\rho_{\delta} v_{\delta}^{3}}=\left[\frac{\alpha(H)}{R_{\delta_{2}}^{0.168}} \frac{\beta(H)}{\alpha(H)} R_{\delta_{2}}^{0.1}\right] \frac{\delta_{?}}{\left(\delta_{2}\right)_{i}}=\frac{\beta(H)}{R_{\delta_{2}}^{0.168}} \frac{\delta_{2}}{\left(\delta_{2}\right)_{i}}
$$

with

$$
\left(\frac{D}{\rho_{\delta} u_{\delta}^{3}}\right)_{i}=\frac{\beta(H)}{R_{\delta_{2}}^{0.168}} \approx \frac{0.0052}{R_{\delta_{2}}^{0.168}}
$$

as the part which represents the dissipation law for the incompressible flow. To be quite correct, a detailed consideration must be made on the definition of the Reynolds number used in eq (69). By definition the dissipation integral expresses the amount of energy dissipated by the viscosity into heat (with the intermediary of turbulence). The related Reynolds number should be more exactly defined with a mean viscosity $\bar{\mu}$ instead of $\mu_{w}$, because the energy transfer by viscosity occurs within the whole boundary layer, although mostly near the wall.

Therefore eq (69) should correctly be written as

$$
\int_{0}^{1} \frac{\tau}{\tau_{w}} d\left(\frac{u}{u_{\delta}}\right)=\frac{\beta(H)}{\alpha(H)}\left(\frac{\mu_{w}}{\bar{\mu}}\right)^{0.1} R_{\delta_{2}}^{0.1}
$$

when the definition (62) for the Reynolds number is used. This correction, however, was proved to be in general so small that it is not essential in the scope of this approximate theory.

\section{d. Simplified Representation for the Wall Friction and Dissipation Law}

The expressions for the incompressible wall friction and for the dissipation law were given by E. Truckenbrodt [4], who evaluated some semiempirical relations found by T. Rotta [3].

The results of the evaluation of $\mathrm{E}$. Truckenbrodt are given in his two figures 3 and 4 which we have reproduced in the present figures 3 and 4 . These have been presented in. order to investigate whether or not it will be possible to find a new suitable fitting for these 


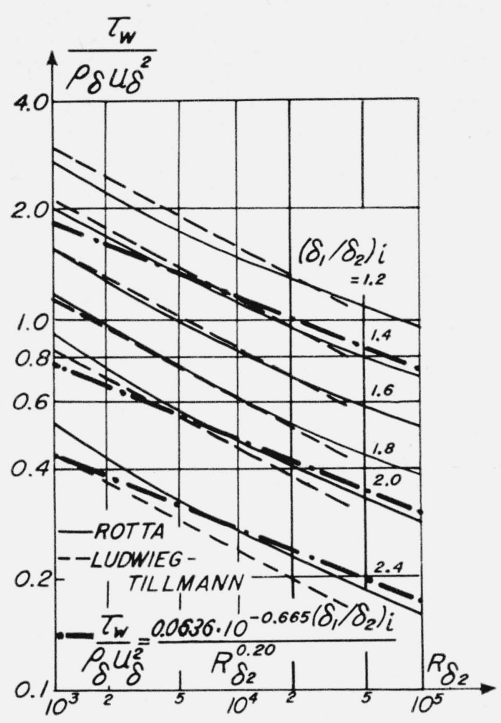

Figure 3. Wall friction law for incompressible turbulent boundary layers.

Thick line: evaluation of experiments of J. Rot.ta-Truckenbrod.

Dashed line: evaluation of experiments of Ludwieg-Tillmann.

Dashed-dotted line: new approximation with 0.2 power law.

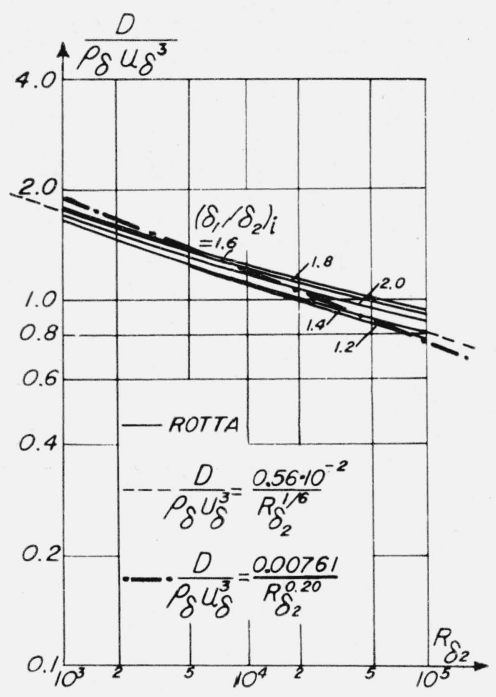

Figure 4. Dissipation law for incompressible turbulent boundary layers.

Thick lines: evaluation of J. Rotta-Trucken. brodt.

Dashed line: approximation proposed by Truckenbrodt.

Dashed-dotted line: new approximation with 0.2 power law.

two empirical laws which permits the use (for the sake of simplification) of only one power of the Reynolds number instead of two (0.268 and 0.168$)$.

We find that if we take the power 0.2 in both cases the fitting of the experimental result in the most important range between $R_{\delta 2}=10^{3}$ and $10^{5}$ will be rather satisfactory. The laws (66) and (70) may then be written as

$$
\begin{gathered}
\frac{\tau_{w}}{\rho_{\delta} u_{\delta}^{2}}=\frac{0.0636 \cdot 10^{-0.665\left(\delta_{1} \delta_{2}\right)_{i}}}{R_{\delta_{2}}^{0.20}} \cdot \frac{\delta_{2}}{\left(\delta_{2}\right)_{i}} \\
\frac{D}{\rho_{\delta} u_{\delta}^{3}}=\frac{0.00761}{R_{\delta_{2}}^{0.20}} \cdot \frac{\delta_{2}}{\left(\delta_{2}\right)_{i}}
\end{gathered}
$$

This simplification prevents the universal function $\Psi_{0}$ of equation (56), from being dependent upon $R_{\delta 2}$ besides $H, M_{\delta}$ and $\theta$. Thus an important simplification in the representation of this function seems possible without noticable loss of accuracy. Detailed investigations on the reliability of this simplification are now in progress.

This simplification will be important if the use of higher momentum equations $(\nu=2,3,4, \ldots$.$) is made. In this case all empirical parts of the wall friction and dissipation$ law are concentrated in the term $\tau_{w} / \rho_{\delta} u_{\delta}^{2}$, and the integrals of type

$$
\int_{0}^{1}\left(\frac{\rho_{\delta}}{\rho}\right)^{\nu} \frac{\tau}{\tau_{w}} d\left(\frac{u}{u_{\delta}}\right) \text { and } \int_{0}^{u_{\delta}}\left(\frac{\rho_{\delta}}{\rho}\right) \nu \frac{\partial}{\partial u}\left[\frac{\tau}{\tau_{w}} \frac{\partial\left(T / T_{\delta}\right)}{\partial\left(u / u_{\delta}\right)}\right] d u
$$

are dependent only upon the shape parameter $H$, the Mach number $M_{\delta}$, and the heat transfer parameter $\theta$. Therefore they can be evaluated, when the family of velocity profiles is chosen. 


\subsection{Evaluation of the Universal Functions of the Two-Equation Method for Laminar and Turbulent Boundary Layers}

The evaluation of the universal functions occurring in the two-equation method as described in section 4 has already been made in the author's publications $[26,27]$ for the case of an insulating wall.

The evaluation of these functions for the case with heat transfer, as suggested by the author, is now under way at the DVL Institute for Applied Mathematics and Mechanics, Freiburg/Br, by considering first the turbulent boundary layer.

A preliminary evaluation for this case with slide rule accuracy has been available for about one year and was used for calculating some illustrating examples which will be discussed in the following section.

This preliminary evaluation showed that the effect of heat transfer on all universal functions can be linearized in a rather good approximation, such that they may be represented in the form

$$
F_{\nu}\left(H, M_{\delta}, \theta\right) \approx F_{\nu}\left(H, M_{\delta} 0\right)+\theta \cdot G\left(H, M_{\delta}\right) .
$$

This result will be checked after the completion of the final evaluation.

\subsection{Examples-Flat Plate}

For the sake of completeness we present here again an important result obtained for the laminar case with insulated wall, figures 5 and 6 . Pohlhausen's method based only upon the momentum integral condition (49) and the wall condition (13) gives $H=1.572=$ const., for the case of $d p / d x=0$ at each Mach number. The consideration of the energy integral

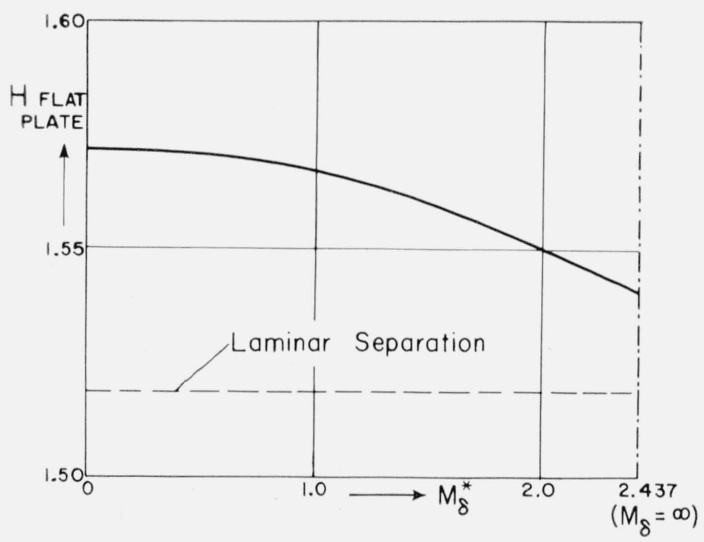

FIGURE 5. Shape parameter $H$ of the laminar velocity profile at a flat plate as a function of the Mach number $M_{\delta}^{*}$.

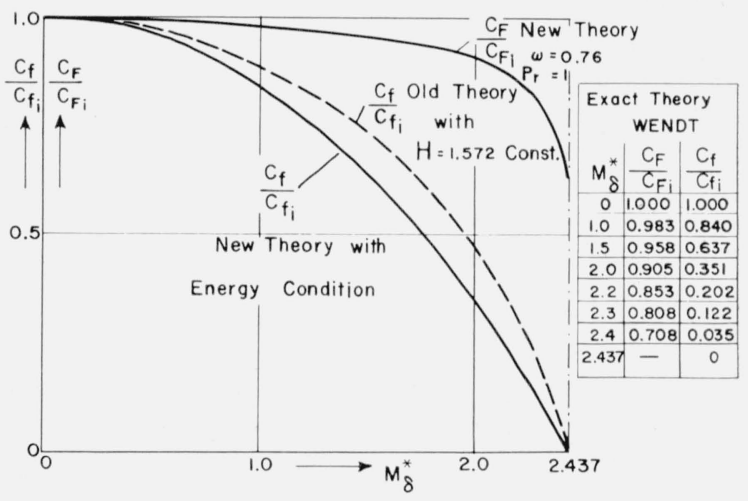

Figure 6. Friction coefficient ratios $c_{f} / c_{f_{i}}$ (local at the same Reynolds number $R_{\delta_{2}}$ ) and $c_{f} / c_{j_{i}}$ (averaged) as a function of the Mach number $M_{\delta}^{*}$.

Laminar boundary layer.

condition (53) instead of eq (13) gives a variation of $H$ with $M_{\delta}^{*}$ and a corresponding change in the behavior of the friction coefficients $c_{f} / c_{f i}$ (local at same values $\mathrm{R}_{\delta_{2}}$ ) and $C_{F} / C_{F i}$ (averaged) with the Mach number $M_{\delta}^{*}$. It is very interesting that the new behavior of $c_{f} / c_{f i}\left(M_{\delta}^{*}\right)$ is almost coincident with the results of an exact theory of $\mathrm{H}$. Wendt [8]. This result shows the effectiveness of the energy integral condition. The related results for the compressible turbulent boundary layer with and without heat transfer are plotted in figures 7 and 8 . The agreement with available experimental data is seen to be good.

Figures 9 and 10 are concerned with the question of how the turbulent separation point in a retarded flow of the type

$$
\frac{u_{\delta}}{u_{\infty}}=\frac{M_{\delta}^{*}}{M_{\infty}^{*}}=1-10 x / l
$$




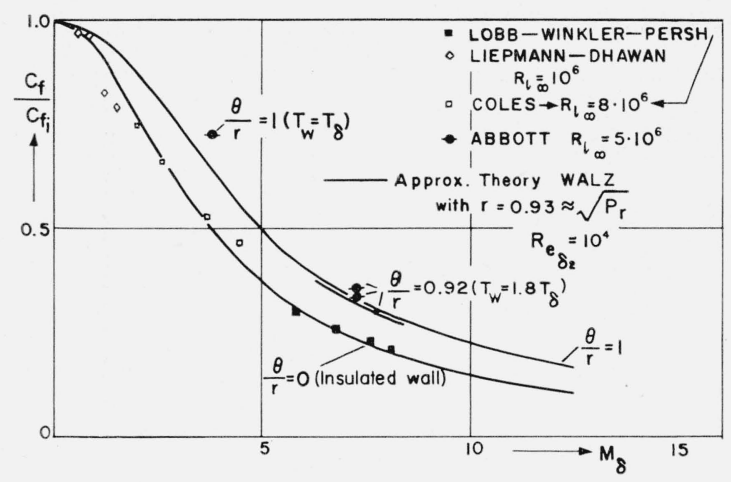

FiguRE 7. Local friction coefficient ratio $c_{f^{\prime}} c_{f_{i}}$ (at the same Reynolds number $R_{\delta_{2}}$ ) of a flat plate as a function of the Mach number $M_{\delta}$ with different values of the heat transfer parameter $\theta / r$.

Tubulent boundary layer.

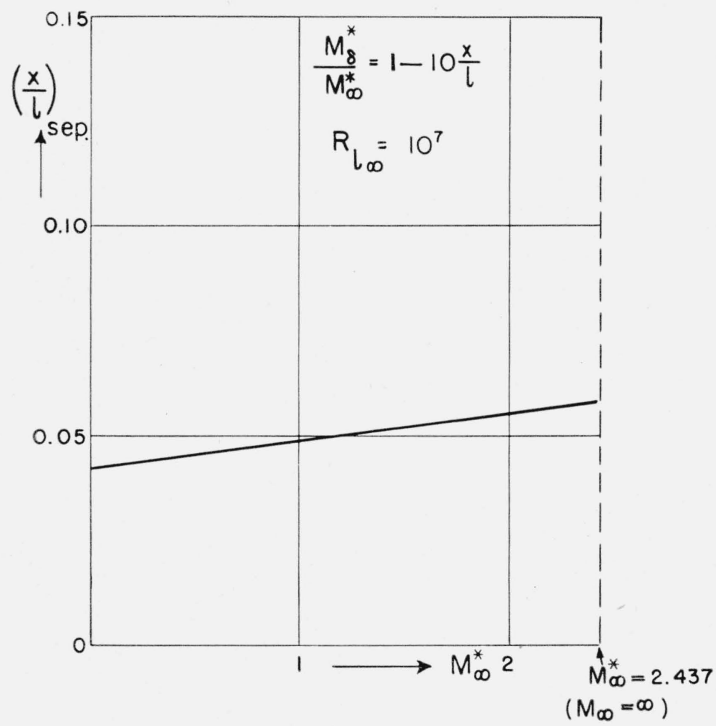

Figure 9. Influence of the Mach number $M_{\infty}^{*}$ on the separation point, insulated wall $(\theta=0)$.

Turbulent boundary layer separation in a retarded flow of the type $\frac{M^{*}}{M_{\infty}}=1-10_{l}^{-}$

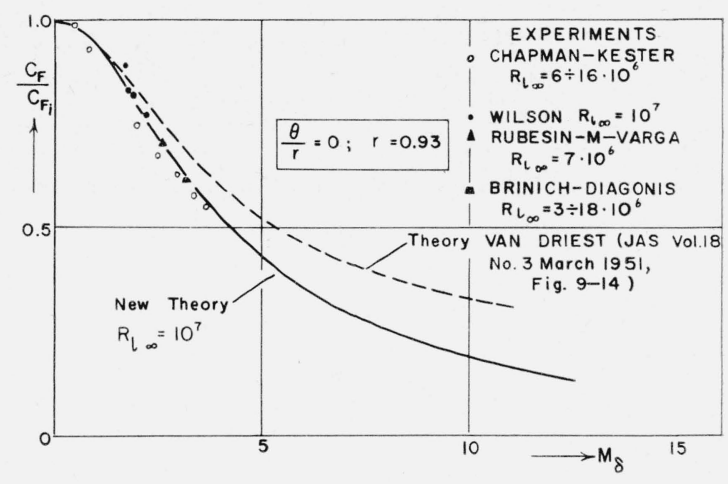

Figure 8. Averaged friction coefficient ratio ${ }^{\circ} c / c_{f_{i}}$ of a flat plate as a function of the Mach number $M_{\delta}$ for insulated wall $(\theta / r=0)$.

Turbulent boundary layer.

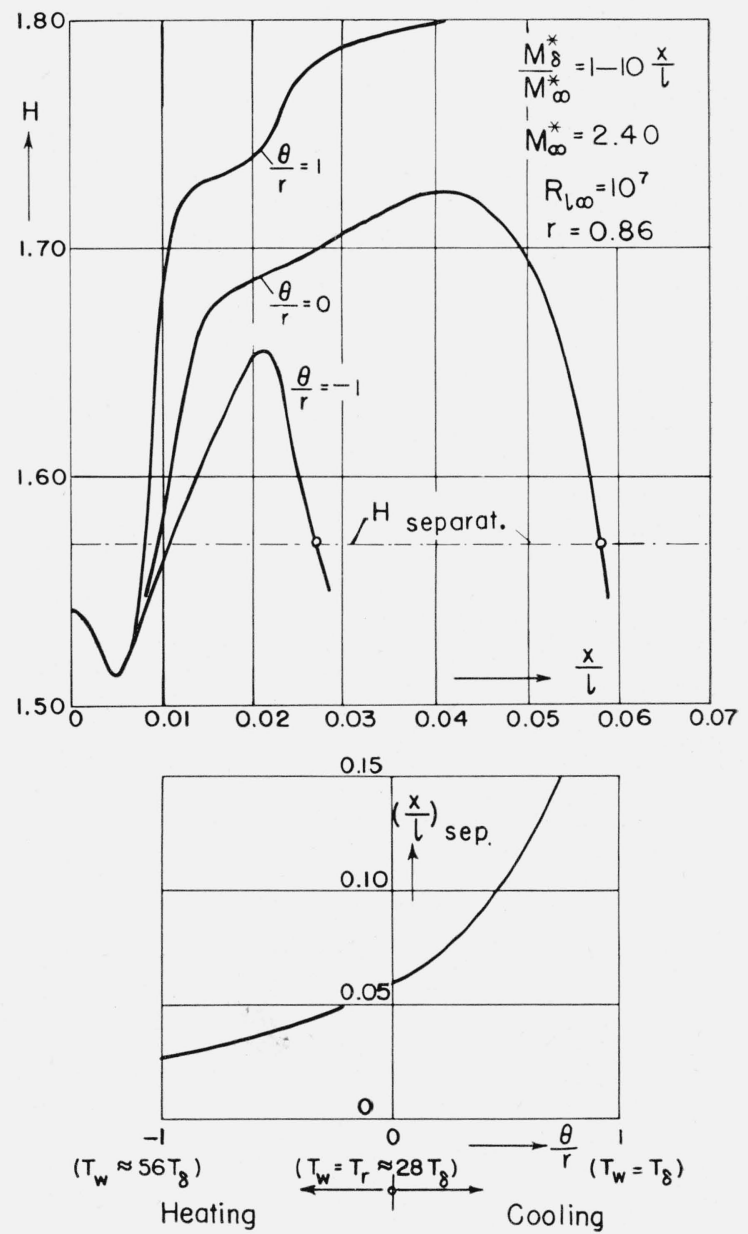

Figures 10 and 11. Influence of heat transfer on the separation point $\left(\frac{x}{l}\right)_{\mathrm{gep}}$ at a constant Mach number $M_{\infty}^{*}=2.40\left(M_{\delta} \approx 12\right)$.

Turbulent boundary layer separation in a retarded flow of the type $\frac{M^{*}}{M_{\infty}}=1-10 \frac{x}{l}$. 
is influenced by the Mach number and by heat transfer. From figure 9 it is seen that the separation point $(x / l)_{\text {sep }}$ is almost independent of variation in the Mach number between $M_{\infty}^{*}=0$ to $2.40\left(M_{\infty}=0\right.$ to 12$)$. Figure 10 shows that by cooling the wall in such a way that the wall temperature $T_{w}$ is kept equal to $T_{\delta}\left(\frac{\theta}{r}=1\right)$, the separation is completely prevented even for the high value $M_{\infty}^{*}=2.40\left(M_{\infty}=12\right)$. On the contrary, heating of the wall moves the separation point upstream. For the theroetical results of the figures 9 and 10, no experimental confirmation is yet available.

\section{Conclusions}

A survey of approximation theories for compressible laminar and turbulent boundary layers with heat transfer at the wall and arbitrary pressure gradient in the flow direction shows that only a two-equation method has been used up to the present, although an infinite number of equations is available. A new derivation for the two infinite systems of integral conditions based upon the partial differential equations for the velocity and temperature field is given.

An investigation of the significance of the equations with higher order shows that their property of describing typical boundary effects vanishes more and more with increasing number $\nu$. Hence, it is not surprising that the results obtained from the two-equation method with $\nu=0$ in both the systems (11) and (40) are good. However, the consideration of one or two equations more would be desirable, at least for a check of the results of the two-equation method in important special cases.

Preliminary investigations have to show how the higher $\nu$-order integral terms containing the shear stress distribution $\tau / \tau_{w}$ can be evaluated in the case of compressible turbulent boundary layers with heat transfer. It has been found that this is possible if the power of the Reynolds number is chosen to be equal for both the wall friction and dissipation law. We have chosen 0.2. In spite of this simplification in these two empirical laws, they still fit the data rather well. Further work on this matter is in progress.

This two-equation method now available with preliminary evaluated universal integral terms has been applied to the study of the dependence of the friction coefficient on compressibility and heat transfer. Good agreement with experimental data has been found.

\section{References}

[1] Th. von Karman, Über laminare und turbulente reibung, Z. Angew. Math. Mech. 1, 233 (1921).

[2] K. Pohlhausen, Zur integration der differentialgleichung der laminaren grenzschicht, Z. Angew. Math. Mech. 1, 252 (1921).

[3] J. Rotta, Schubspannungsverteilung und energie dissipation bei turbulenten grenzschichten, Ingr.-Arch. 20, 195 (1952).

[4] E. Truckenbrodt, Ein quadraturverfahren zur berechnung der laminaren und turbulenten reibungsschicht bei ebener und rotations symmetriseher strömung, Ingr.-Arch. 20, 212 (1952).

[5] A. Busemann, Gasströmung mit laminarer grenzschicht entlang einer Platte, Z. Angew. Math. Mech. 15, 23 (1935).

[6] D. R. Chapman, W. M. Rubesin, Temperature and velocity profiles in the compressible laminar boundary layer with arbitrary distribution of surface temperature, J. Aeronaut. Sci. 16, 547 (1949).

[7] L. Croceo, Sullo strato limite laminare nei gas lungo una lamina plana. Rend. Math. Univ. Roma 5, 2 (1951).

[8] H. Wendt, Die kompressible laminare grenzschicht an der ebenen platte und am kreiskegel, DEFA-LBRABericht, 2 (St. Louis, Elsass, 1947).

[9] L. S. Leibenson, Energy form of the integral conditions in the boundary layer theory CAHI Rept. No. 240, pp. 41-44 (1935).

[10] Golubew, Theoretical hydromechanics, Part II. J. A. Kihel, W. E. Xochin and N. B. Rose, eds. (State Publishing House for Technical \& Theoretical Literature, Moscow, 1957). pp. 404-407.

[11] G. L. Sutton, An approximate solution of the boundary layer equations for a flat plate, Phil. Mag. [7], 23 1,146 (1937).

[12] K. Wieghardt, On an energy equation for the calculation of laminar boundary layers. B.J.G.S. 65, Joint Jubell. Object. Agency. (July 31, 1946). 
[13] L. E. Kalikhman, Heat transmission in boundary layer, Nat. Advisory Comm. Aeronaut. Tech. Note $1229(1949)$.

[14] P. A. Libby, M. Morduchow, and M. Bloom, Critical study of integral methods in compressible laminar boundary layers, Nat. Advisory Comm. Aeronaut. Tech. Note 2655 (1952).

[15] D. N. Morris and J. W. Smith, The compressible laminar boundary layer with arbitrary pressure and surface temperature gradients, J. Aeronaut. Sci. 20, 805 (1953).

[16] A. Walz, Anwendung des energiesatzes von Wieghardt auf einparametrige geschwindigkeits profile in laminaren grenzschichten, Ingr.-Arch. 16, 243 (1948).

[17] E. Gruschwitz, Die turbulente reibungsschicht in ebener strömung bei druckabfall und druckanstieg, Ingr.-Arch. 2,321 (1931).

[18] H. Ludwieg and W. Tillmann, Untersuchungen über die wandschubspannung in turbulenten reibungsschichten, Ingr.-Arch. 17, 288 (1949).

[19] A. Walz, Ein neuer ansatz für das geschwindigleitsprofil der laminaren reibungschicht, Lilienthal-Bericht No. 141, 8, 1941 der deutschen Luftfahrt-Forschung.

[20] R. Sauer, Gasdynamik, einfuhrung in die theorie, 2d Ed. (Springer, Berlin Göttingen Heidelberg, 1951).

[21] H. Schlichting, Boundary layer theory (McGraw-Hill Book Co., Inc., New York, N.Y., 1955).

[22] E. R. van Driest, On the boundary layer with variable Prandtl number (North American Aviation Inc., Calif., 1954).

[23] L. Prandtl, Führer durch die strömungslehre, (Friedr. Vieweg \& Sohn, Braunschweig, 1957).

[24] F. H. Clauser, Turbulent boundary layers in adverse pressure gradients, J. Aeronaut. Sci. 21, 91 (1954).

[25] R. E. Wilson, Heat transfer and skin friction investigations in the NOL hypersonic wind tunnel, Rept. of the U.S. Naval Ordnance Lab., White Oak, Silver Spring, Md. (1955).

[26] A. Walz, New calculation method for laminar and turbulent boundary layers in compressible flow (Publications Scientifiques et Techniques du Ministere de l'AIR, No. 309 (1956) and No. 336 (1957) Paris, France).

[27] A. Walz, Näherungstheorie für kompressible turbulente grenzschichten, Z. Angew. Math. Mech. Sonderheft, S50 (1956).

Washington, D.C.

(Paper 63B1-8) 


\section{Publications of the National Bureau of Standards}

\section{Selected Abstracts}

Mechanized computation of thermodynamic tables at the National Bureau of Standards, J. Hilsenrath and J. H. Wegstein, Thermodynam. Properties of Fluids, London, July 10-12, 1957, Proc. Inst. of Mech. Engrs. (London) (1958).

A high speed computing routine is described which computes the chemical composition and certain thermodynamic properties of homogeneous gaseous systems containing up to 5 elements. The procedure, which is applicable over a wide temperature range in which both dissociation and ionization effects are allowed for, permits the computation of mole fractions, compressibility factor, internal energy, enthalpy and entropy of the equilibrium mixtures at specified temperatures and at specified densities or pressure intervals. Auxiliary routines for the IBM 704 are used to compute the specific heats, specific heat ratios, isentropic expansion coefficient and the sound velocity. A brief description is given of the library of thermodynamic tables that provides the input data for these calculations. An appendix to this paper gives an indexed bibliography of existing thermodynamic functions for the products of a wide variety of combustion reactions.

Further identities and congruences for the coefficients of modular forms, M. Newman, Can. J. Math. 10, $57 \%(1958)$.

Let $p_{r}(n)$ be the coefficient of $x^{n}$ in $\Pi\left(1-x^{n}\right)^{r}$. Let $r$ be odd, $0<r<24$. Suppose $p$ is a prime such that $p>3$ when $(r, 3)=1$ and $p>2$ when $3 \mid r$. Then it is shown that for all integral $n$,

$$
p_{r}\left(n p^{2}+r \nu\right)-\gamma_{n} p_{r}(n)+p^{r-2} p_{r}\left(\frac{n-r \nu}{p^{2}}\right)=0,
$$

where $\gamma_{n}=c-\frac{(r \nu-n)}{p} p^{(r-3) / 2} \quad a$, and $c$ and $a$ are certain constants.

A fractional factorial experiment of the mixed $2^{m} 3^{n}$ series, W. S. Connor, Proc. 14th Ann. Clin. Rochester Soc. for Quality Control (Feb. 1958).

The paper is concerned with a fractional factorial design for three factors each of which has two levels and two factors each of which has three levels. The complete factorial consists of 72 treatment combinations but the fractional factorial of only 36 treatment combinations. An example is worked out.

A note on confidence intervals in regression problems J. Mandel, Ann. Math. Statist. No. 3, 29, 892 (Sept. 1958).

A method is presented for the construction of jointly valid confidence intervals for arbitrary real functions of the coefficients of a multiple linear regression. In the case of a single independent variable, $x$, the problem of constructing jointly valid confidence intervals for the $\mathrm{x}$-values corresponding to a set of "future" measurements of the dependent variable is also discussed.

Harmonic generation with ideal rectifiers, C. H. Page, Proc. I.R.E., No. 10, 46, 1738 (Oct. 1958).

It is shown that the $n$th harmonic cannot be generated with an efficiency exceeding $1 / n^{2}$. Of the power converted to dc and harmonics, at least 75 percent is de dissipation, and this cannot be reduced by an arrangement of selective circuits.
Two forms of mathematical induction, A. Schach, Math. Mag., No. 2, 32, 83 (Nov.-Dec. 1958).

It is shown that two principal forms of (finite) mathematical induction:

(I) If $P(1)$ and if, for all pos. int. $n, P(n)$ implies $P(n+1)$, then $P(n)$ for all pos. int. $n$; and

(II) If $P(1)$ and if, for all pos. int. $n, P(1) \& P(2) \& \ldots \& P(n)$ implies $P(n+1)$, then $P(n)$ for all pos. int. $n$

are equivalent; and that any demonstration based on one of them can be recast so that it is based on the other instead.

The evaluation of matrix inversion programs, M. Newman and J. Todd, J. Soc. Indust. Appl. Math., No. 4, 6, 466 (Dec. 1958).

A set of thirteen test matrices typical of those occurring in practice were inverted on SEAC, the 704, and various other machines. The results were compared with the theoretical inverses and various error estimates were computed.

Un nouveau critere d'univalence des transformations dans un $R^{n}$, M. A. Ostrowski, Compt. rend. acad. sci. 248, 348 (Jan. 1959).

A criterion of univalence of an $n$-dimensional transformation, given in a former communication, is generalized; the possibility of further generalizations and refinements is discussed.

On the convergence of the Rayleigh quotient iteration for the computation of the characteristic roots and vectors. II, A. M. Ostrowski, Arch. Rational Mech. Anal. No. 5, 2, 423 (1959).

Further asymptotic properties of the Rayleigh quotient algorthm are derived, and numerical results for some special matrices are given.

A note of the computation of $x^{2}, \mathrm{M}$. G. Natrella, Am. Statistician, No. 1, 13, 20 (Feb. 1959).

There is a useful, but not widely-known short cut available for computing $\chi^{2}$ for the comparison of two observed frequency distributions with equal total counts. The advantages are that it does not require computing the expected frequencies, avoids problems of rounding the deviations, and requires only one division per class instead of one division per cell. It can be extended to three observed distributions, but loses its computational advantages for more than three.

Mode expansion in the low-frequency range for propagation through a curved stratified atmosphere, H. Bremmer, J. Research NBS 63D, No. 1, 75 (1959).

This expansion is particularly useful when considering ionospheric propagation at low frequencies. The complex problem dealing with two media, viz. a homogeneous earth and a surrounding stratified atmosphere, leads to intractable expressions. However, as the influence of the earth may be accounted for by an approximate boundary condition at the earth's surface, the problem is then reduced to that of the outer medium only. The coefficients of the mode expansion for this simplified problem will be derived while taking into account the earth's curvature; however, the latter proves to be negligible under very general conditions. The expansion to be derived is wanted in particular when studying the influence of a gradual transition in the electron density with height at the lower edge of the ionosphere. 
On the theory of fading properties of a fluctuating signal imposed on a constant signal, $H$. Bremmer, NBS Circ. 599 (May 1959).

This Circular is a theoretical mathematical consideration of the varying strength of a radio signal consisting of two components: a constant signal, and a fluctuating signal of the same frequency which has reached the receiver by a different path. The properties investigated for the composed signal are the distribution functions of both the amplitude and phase, as well as the average number of crossings for either quantity through any given level.

The first part of this Circular deals with the idealized case, where the fluctuating signal is considered to be "random." The second part is a more practical consideration of the characteristics of the composite signal when the fluctuating signal is a "quasi-random" result of first order scattering in a turbulent atmosphere. In the two extreme cases, where the received signal is composed entirely of either the constant, or line-of-sight, signal, or the fluctuating, reflected signal, the complicated general formulas are simplified.

This Circular will be of interest to those who deal with the various types of equipment and effects which pertain to the fading properties of this composite signal.

\section{The mean deviation of the Poisson distribution,} E. L. Crow, Biometrika, pts. 3 and 4 45, 556-55.9 (Dec. 1958).

The mean deviation of the Poisson distribution is easily determined to be $2 m Y$, where $m$ is the mean of the distribution and $Y$ is the maximum value of the individual Poisson probabilities. The ratio, $R(m)$, of the mean deviation to the standard deviation is shown to be a damped oscillation as a function of $m$, converging rapidly toward the limiting normal value $\sqrt{2 / \pi}$. Furthermore $R(m)$ takes on the normal value twice between every pair of successive non-negative integers. Thus $R(m)$ is a poor criterion for distinguishing the Poisson and normal distributions.

A property of additively closed families of distributions, E. L. Crow, Ann. Math. Stat. No. 3, 29, 892-897 (Sept. 1958).

Consider a one-parameter additively closed family of univariate cumulative distribution functions $F(x ; \lambda)$ (H. Teicher, Ann. Math. Stat., Vol. 25 (1954), pp. 775-778). Let three cumulants with orders in arithmetic progression exist and be nonzero. If all three orders are even, or if the first order is odd, it is also required that $F(x ; \lambda)=0$ for $x<0$ and $F(x ; \lambda)$ $>0$ for $x>0$. Consider linear combinations, with real, nonzero coefficients, of a finite number of independent variables with distributions in the family. It is proved that the only such linear combinations whose distributions are also in the family are those with coefficients unity. The additively closed families having this property may be called strictly additively closed. It can be shown that (one-parameter) additively closed stable families of distributions (normal and Cauchy in particular) with characteristic functions continuous in $\lambda$ are not strictly additively closed, while Poisson, generalized Poisson, binomial, and gamma families are strictly additively closed.

\section{Other NBS Publications}

Journal of Research Section A. Physics and Chemistry, Volume 63A, No. 1, July-August 1959. 70 cents. A pproximate issue date, August 1, 1959.

Description and analysis of the first spectrum of iodine. C. C. Kiess and C. H. Corliss.

$\mathrm{CH}$ in the solar spectrum. Charlotte E. Moore and Herbert P. Broida.

Infrared studies in the 1 - to 15 -micron region to 30,000 atmospheres. C. E. Weir, E. R. Lippincott, A. Van Valkenburg, and E. N. Bunting.

Phosphinoborine compounds: mass spectra and pyrolysis. Leo A. Wall, Sidney Strauss, Roland E. Florin, Fred L. Mohler, and Paul Bradt.

Experimental and theoretical study of kinetics of bulk crystallization in poly(chlorotrifluoroethylene). John D. Hoffman, James J. Weeks, and W. M. Murphey,

Journal of Research Section C. Engineering and Instrumentation, Volume 63C, No. 1, July-September 1959. 75 cents. Approximate issue date, August 15, 1959.

Creep of cold-drawn nickel. William D. Jenkins and Carl R. Johnson.

Friction and endurance of prelubricated and unlubricated ball bearings at high speeds and extreme temperatures. Hobart S. White.

Effect of light and water on the degradation of asphalt. L. R. Kleinschmidt and H. R. Snoke.

Current and potential relations for the cathodic protection of steel in a high resistivity environment. W. J. Schwerdtfeger.

A tilting air-lubricated piston gage for pressures below onehalf inch of mercury. U. O. Hutton.

Compact multianvil wedge type high-pressure apparatus. E. C. Lloyd, U. O. Hutton, and D. P. Johnson.

A coulometric-titration coulometer. Stanley W. Smith and John K. Taylor.

Electron beam magnetometer. L. Marton, Lewis B. Leder, J. W. Coleman, and D. C. Schubert.
A refined X-band microwave microcalorimeter. Glenn F. Engen.

Journal of Research Section D. Radio Propagation, Volume 63D, No. 1, July-August 1959. 70 cents. Approximate issue date, July 15, 1959.

Preliminary results of the National Bureau of Standards radio and ionospheric observations during the International Geophysical Year. David M. Gates.

Origin of [OI] 5577 in the airglow and the aurora. Franklin E. Roach, James W. McCaulley, and Edward Marovich.

Comparison of absolute intensities of [OI] 5577 in the auroral and subauroral zones. F. E. Roach, J. W. McCaulley, and C. M. Purdy.

Origin of "very low-frequency emissions." R. M. Gallet and R. A. Helliwell.

Climatology of ground-based radio ducts. Bradford R. Bean. Power requirements and choice of an optimum frequency for a worldwide standard-frequency broadcasting station. A. D. Watt and R. W. Plush.

Measurements of phase stability over a low-level tropospheric path. M. C. Thompson, Jr. and H. B. Janes.

System loss in radio wave propagation. Kenneth A. Norton.

Mode expansion in the low-frequency range for propagation through a curved stratified atmosphere. H. Bremmer.

Transmission and reflection by a parallel wire grid. Martin T. Decker.

Synoptic variation of the radio refractive index. B. R. Bean and L. P. Riggs.

Low-frequency propagation paths in arctic areas. A. D. Watt, E. L. Maxwell, and E. H. Whelan.

A property of additively closed families of distributions, E. L. Crow, Ann. Math. Statist. No. 3, 29, 892 (Sept. 1958).

The mean deviation of the Poisson distribution, E. L. Crow, Biometrika 45, 556 (1958). 